\title{
SISPRED 2021
}

XIV Simpósio Nacional de Sistemas Prediais

Gestão, Eficiência e Sustentabilidade

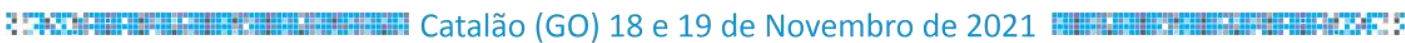

\section{ESTUDO BIBLIOMÉTRICO DA LITERATURA SOBRE O SISTEMA SIFÔNICO DE DRENAGEM DE ÁGUAS PLUVIAIS}

\section{Bibliometric Study of Literature on Siphonic Rainwater Drainage System MORGADO, Uiara Talitta Martins Araújo ${ }^{1}$ CAMPOS, Marcus André Siqueira ${ }^{2}$}

Recebido em 16 de julho de 2021, aprovado em 06 de setembro de 2021, publicado em 18 de novembro de 2021

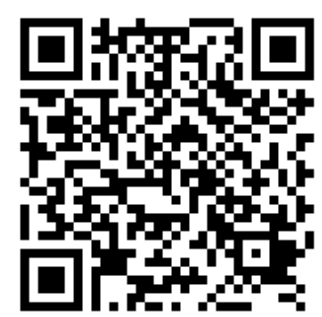

Palavras-chave:

Sistema Sifônico de Drenagem de Águas Pluviais;

Mapeamento da literatura;

Bibliometria;

VosViewer.

Keywords:

Siphonic Rainwater Drainage System; Literature mapping; Bibliometrics; VosViewer.
RESUMO: Este artigo teve como objetivo realizar um mapeamento da Literatura sobre a produção bibliográfica do Sistema Sifônico De Drenagem De Águas Pluviais, a fim de que sejam identificadas tanto áreas de maior número de estudos, quanto as áreas pouco exploradas. Foi realizada uma busca de artigos publicados nas bases de dados Engineering Village, Science Direct, Scopus e Web of Science, por meio de critérios pré-definidos e um total de 24 artigos aderentes foram selecionados. Com as análises realizadas, são ofertadas recomendações para futuros estudos e uma visão geral da literatura sobre o conteúdo.

ABSTRACT: This article aimed to map the literature on the bibliographic production of the Siphonic Stormwater Drainage System, in order to identify the areas with the greatest number of studies and the underexplored areas. A search for articles published in the Engineering Village, Science Direct, Scopus and Web of Science databases was performed using predefined criteria and a total of 24 adherent articles were selected. With the analyzes carried out, recommendations for future studies and an overview of the literature on the content are offered.

\section{CONTATO DOS AUTORES:}

${ }^{1}$ MORGADO, Uiara Talitta Martins Araújo, Mestranda em Construção Civil, PPG-GECON- Escola de Engenharia Civil e Ambiental da Universidade Federal de Goiás (EECA/UFG), uiaramorgado@discente.ufg.br .

2 CAMPOS, Marcus André Siqueira, Professor Doutor da Universidade Federal de Goiás, PPG-GECONEscola de Engenharia Civil e Ambiental da Universidade Federal de Goiás (EECA/UFG), marcus_campos@ufg.br. 


\section{INTRODUÇÃO}

Os sistemas de drenagem ditos convencionais necessitam do ar para o seu funcionamento, uma vez que nestes sistemas a água escoa por gravidade e o sistema opera unicamente sob pressão atmosférica (ARTHUR E SWAFFIELD, 1999).

Para grandes coberturas $\left(>5.000 \mathrm{~m}^{2}\right)$, em sistemas de drenagem convencionais com calhas internas, faz-se necessário a utilização de caixas de passagem dentro da edificação, os condutores horizontais no sistema necessitam de inclinação, é preciso também a utilização de várias colunas com diâmetros maiores, estes fatos geram tanto redução na capacidade do sistema, como interferências arquitetônicas e para a redução destes é preciso utilizar técnicas mais modernas, já disponíveis no mercado.

Uma alternativa consiste no Sistema Sifônico de Drenagem de Água Pluvial (SSDAP), sistema este que trabalha sob pressão. $O$ ar é impossibilitado de entrar na tubulação devido aos captores tipo antivórtice, fazendo com que a tubulação trabalhe a seção plena.

A maior capacidade de drenagem dos SSDAP, permite que ocorram: reduções nos diâmetros, menor tempo de execução da rede, eliminação de declividades, redução na quantidade de prumadas e diminuição das interferências arquitetônicas, os tornando assim atraentes para grandes coberturas (MAY, 2004).

Mesmo com sua crescente utilização, poucos são os trabalhos voltados a análise da literatura no tocante ao funcionamento do sistema e com a proposta de compreender melhor o que a bibliografia traz sobre o assunto. Assim, este artigo realizou um mapeamento de literatura sobre a produção bibliográfica do SSDAP, a fim de que fossem identificadas tanto áreas de maior número de estudos, quanto as áreas pouco exploradas, entendendo assim o estágio geral de desenvolvimento das pesquisas sobre o tema.

\section{MATERIAIS E MÉTODOS}

Para a elaboração do mapeamento, foram seguidas as diretrizes propostas por Kitchenham (2004) sendo realizada a divisão do estudo em três estágios, conforme processo demonstrado na Tabela 1.

Tabela 1 - Diretrizes para mapeamento da literatura

\begin{tabular}{|c|c|l|}
\hline Etapas & \multicolumn{1}{|c|}{ Estágios } & \multicolumn{1}{|c|}{ Descrição } \\
\hline Etapa i & Planejamento da Revisão & $\begin{array}{l}\text { Identificação da questão de pesquisa } \\
\text { Protocolo da revisão }\end{array}$ \\
\hline Etapa ii & Condução da Revisão & $\begin{array}{l}\text { Identificação da pesquisa } \\
\text { Seleção de estudos } \\
\text { Avaliação da Qualidade } \\
\text { Extração e monitoramento dos dados } \\
\text { Síntese dos dados }\end{array}$ \\
\hline Etapa iii & Documentação da Revisão & $\begin{array}{l}\text { Analise Bibliométrica dos resultados } \\
\text { Síntese e documentação dos resultados }\end{array}$ \\
\hline
\end{tabular}

Fonte - Adaptado de Kitchenham (2004). 


\subsection{Planejamento da Revisão}

Identificação da questão de pesquisa

A questão de pesquisa que norteou o mapeamento foi: Qual o panorama da produção científica sobre o SSDAP?

\section{Protocolo da revisão}

Com o intuito de responder à pergunta de pesquisa, elaborou-se um protocolo de revisão, tendo como população de pesquisa todos os artigos que contemplem o funcionamento do SSDAP e foram excluídos os artigos que trouxessem tratativas sobre o sistema de drenagem convencional de águas pluviais.

Os termos de pesquisa articulados (strings) utilizadas foram: "Siphonic Drainage Pluvial" OR "Siphonic Outlets " OR "Siphonic Roof Drainage System" no intuito de abarcar o maior número de documentos que tratassem do assunto, não deixando nenhum trabalho relevante de fora da seleção.

Optou-se em buscar apenas documentos que tivessem sido publicados em periódicos internacionais. Para tal, selecionou-se bases internacionais de relevância no cenário científico mundial. As bases selecionadas foram: Engineering Village, Science Direct, Scopus e Web of Science por serem estas as bases que contém um maior número de pesquisas representativas para a engenharia.

\subsection{Condução da Revisão}

Identificação da pesquisa /seleção dos estudos

Considerou-se como parâmetro apenas artigos publicados de 1990 em diante e com o intuito de refinar a busca foi adotado o critério de seleção de apenas artigos na língua inglesa.

Todos os documentos obtidos neste estágio foram importados para o website Parsifal (https://parsif.al) e os estudos nele gerenciados.

\section{Seleção dos artigos}

Foram analisados manualmente cada estudo e excluídos os trabalhos que estivessem duplicados e que não se enquadravam como descrito na população do planejamento.

Exclusões foram feitas através das etapas: leitura dos títulos, resumos e leitura de artigos na íntegra, eliminando assim, os não aderentes.

\section{Extração e monitoramento dos dados}

Após exportados do Parsifal e dispostos em uma planilha eletrônica, foram extraídos de cada estudo os seguintes dados: A fonte (revista ou conferência) e referência completa, ano de publicação, autores e sua instituição e o país onde se encontra situado, referências e palavras chaves.

\section{Síntese dos dados}

A síntese de dados envolve a comparação e o resumo dos estudos, que para Kitchenham (2004) esta etapa tem o fim de gerar um novo conhecimento a partir dessas análises. 
Os dados foram tabulados para mostrar as técnicas utilizadas em cada trabalho e realizada uma classificação do tipo de trabalho (estudo de caso, teórico, experimental).

\subsection{Documentação da revisão}

A etapa de documentação objetivou descrever os artigos selecionados por meio de uma análise bibliométrica, onde foram selecionados como indicadores: A quantidade de artigos publicados por ano, os autores mais citados, fontes das publicações, fator de impacto, principais palavras chaves.

Para as análises de redes como os autores mais frequentes, cocitação de autores, cocitação de referência e coocorrência de palavras chaves, utilizou-se o programa computacional VOSviewer. Esta ferramenta é fundamentada na construção de mapas baseados nas distâncias e em gráficos, conforme explanado por Jan van Eck, et al. (2009).

\section{RESULTADOS}

As buscas nas bases de dados retornaram um total de 103 trabalhos, sendo estes, distribuídos conforme diagrama de Venn apresentado na Figura 1. A base que apresentou um maior número de artigos foi a Science Direct contendo 61\% dos trabalhos, seguida da base Scopus com 27,4\% e as bases Engineering Village e Web of Science igualmente com 5,8\%.

\section{Figura 1 - Diagrama de Venn}

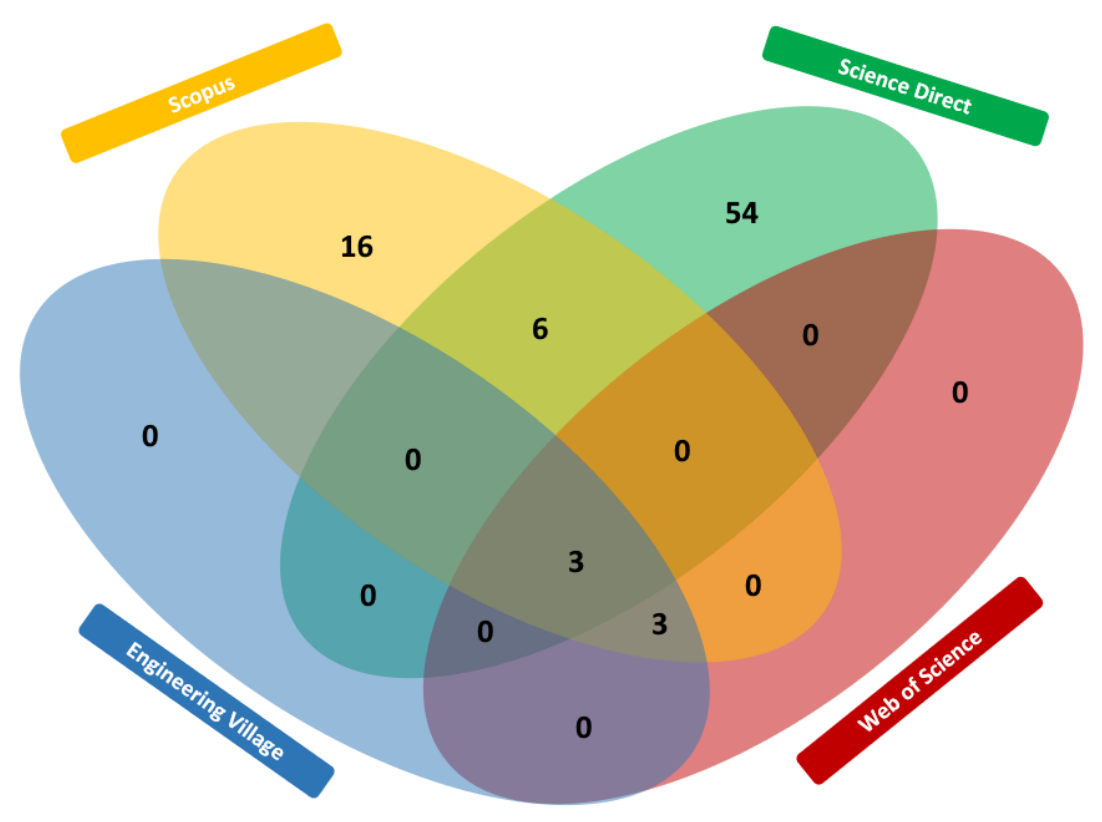

Fonte: Elaborada pelos Autores (2021).

Após a seleção dos artigos, iniciou-se a exclusão dos mesmos. Do total de artigos, verificouse que 23 estavam duplicados. Eliminou-se mais 52 artigos por leitura de títulos e mais 2 por leitura de resumos e 2 por leitura dos textos completos. Não se obteve nenhum artigo por meio da amostragem bola de neve (snowball sampling). Desta forma, 24 artigos foram analisados. 
A primeira análise realizada foi sobre os anos de publicação, demostrada na Figura 2. Percebeu-se que não houve grandes variações durantes os anos, destaque para o ano de 2010 e 2014 com maior número de publicações.

Figura 2 - Classificação por publicações por ano

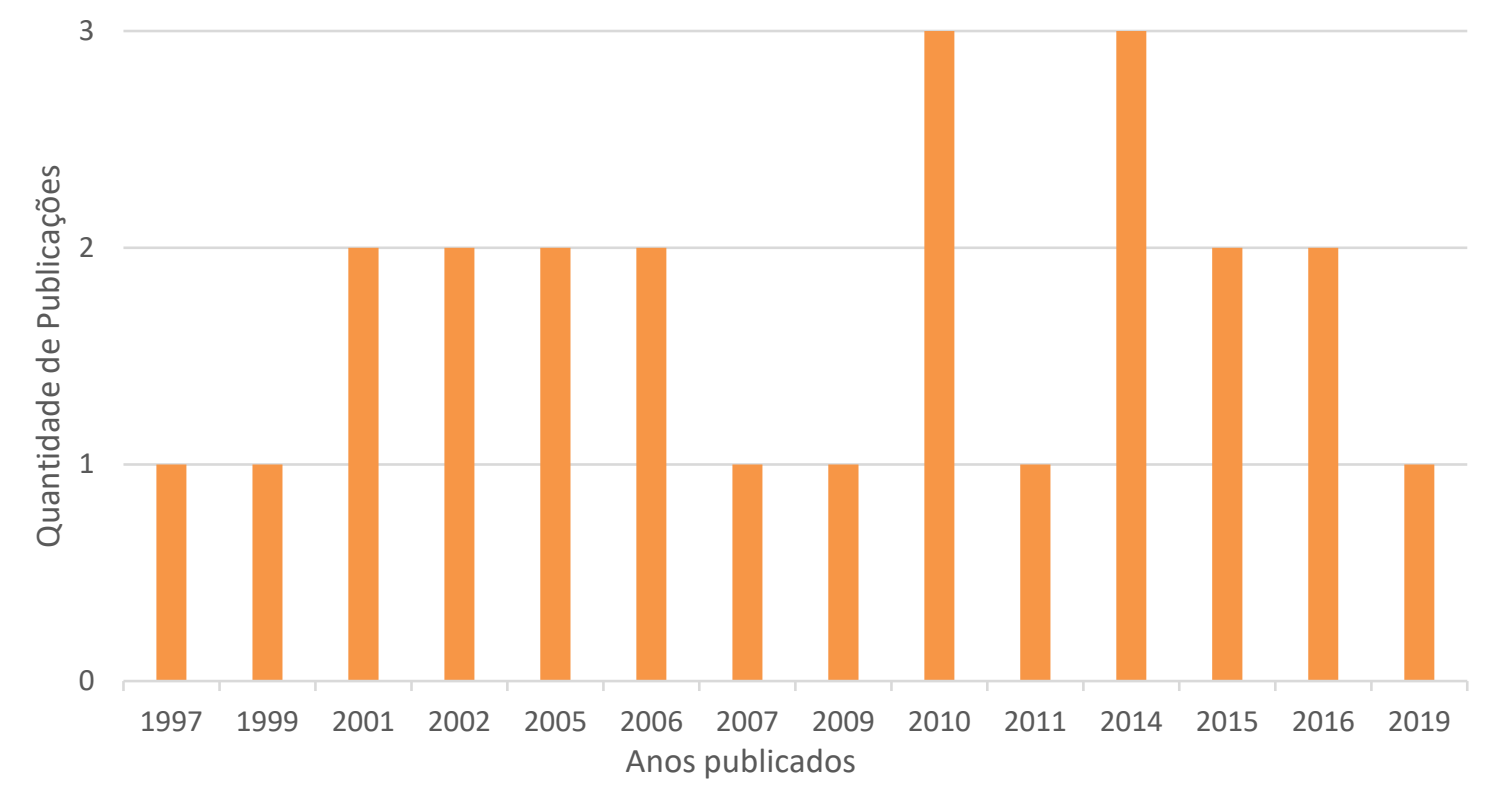

Fonte: Elaborada pelos Autores (2021).

Outra análise foi o local de publicação dos artigos bem como o fator de impacto dos mesmos. Neste trabalho, utilizou-se o Journal Citation Report (JCR). Eles são apresentados na Figura 3. Os documentos apresentaram uma variedade na qualidade dos periódicos, indo de 0,419 e atingindo um máximo de 4,971 em cinco publicações.

Figura 3 - Classificação por meio de publicação

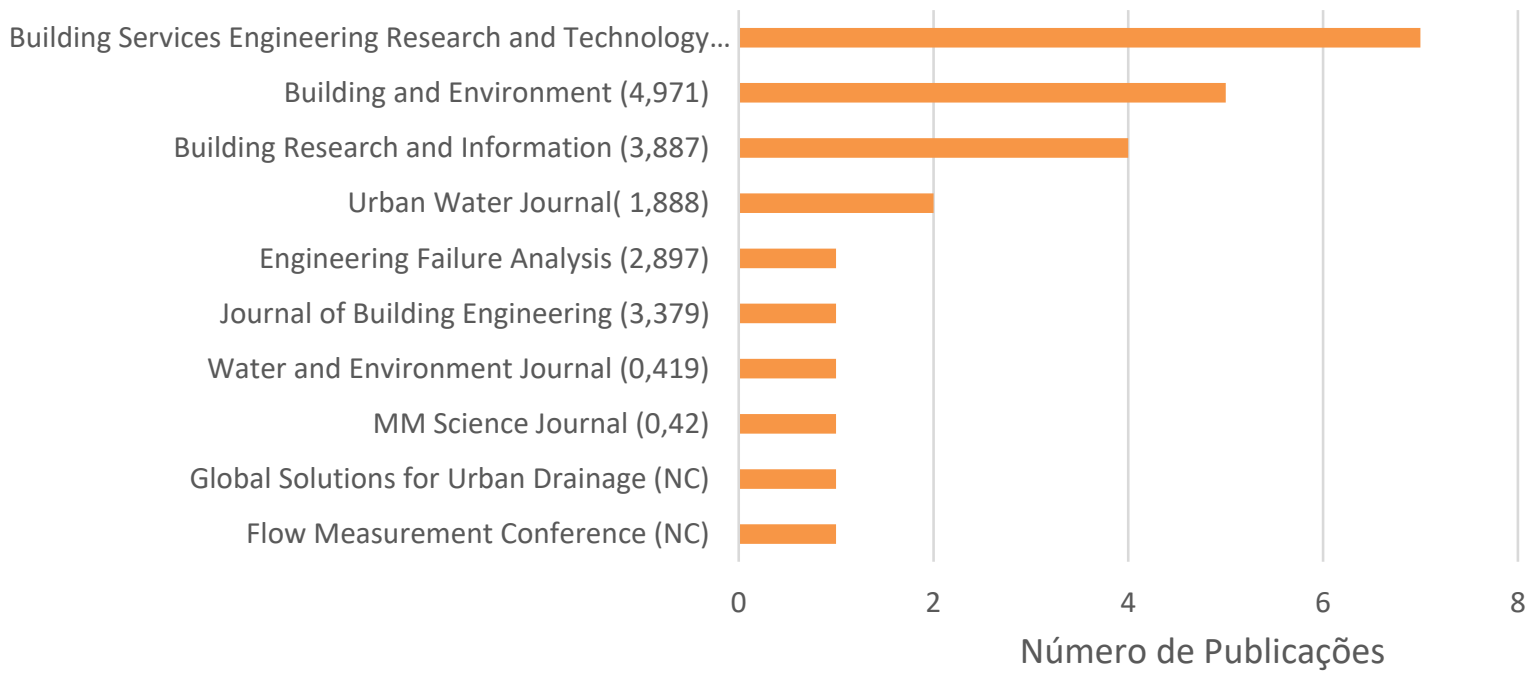

Fonte: Elaborada pelos Autores (2021). 
A Figura 4, mostra a análise de rede da colaboração de coautoria entre os 14 autores mais relevantes.

Em destaque tem-se os autores Swaffield J.A, seguido de Arthur s. e Wright G.B., com maior número de citações.

Figura 4 - Mapa de Coautoria (Autores)

campbell

fucik d.

mayer.w.

ozakgul

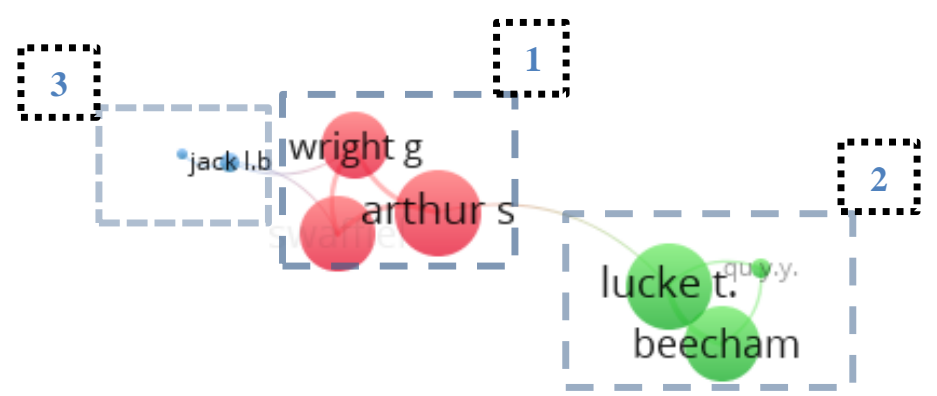

Fonte: Elaborada pelos Autores (2021).

A Figura 5 mostra a rede de relacionamentos de cocitação de autores. $O$ tamanho de cada nó indica a quantidade de citações que determinado autor recebeu. Quanto mais próximos dois nós estão, mais forte é a relação de cocitação entre eles.

Figura 5 - Mapa de Cocitação de Autores

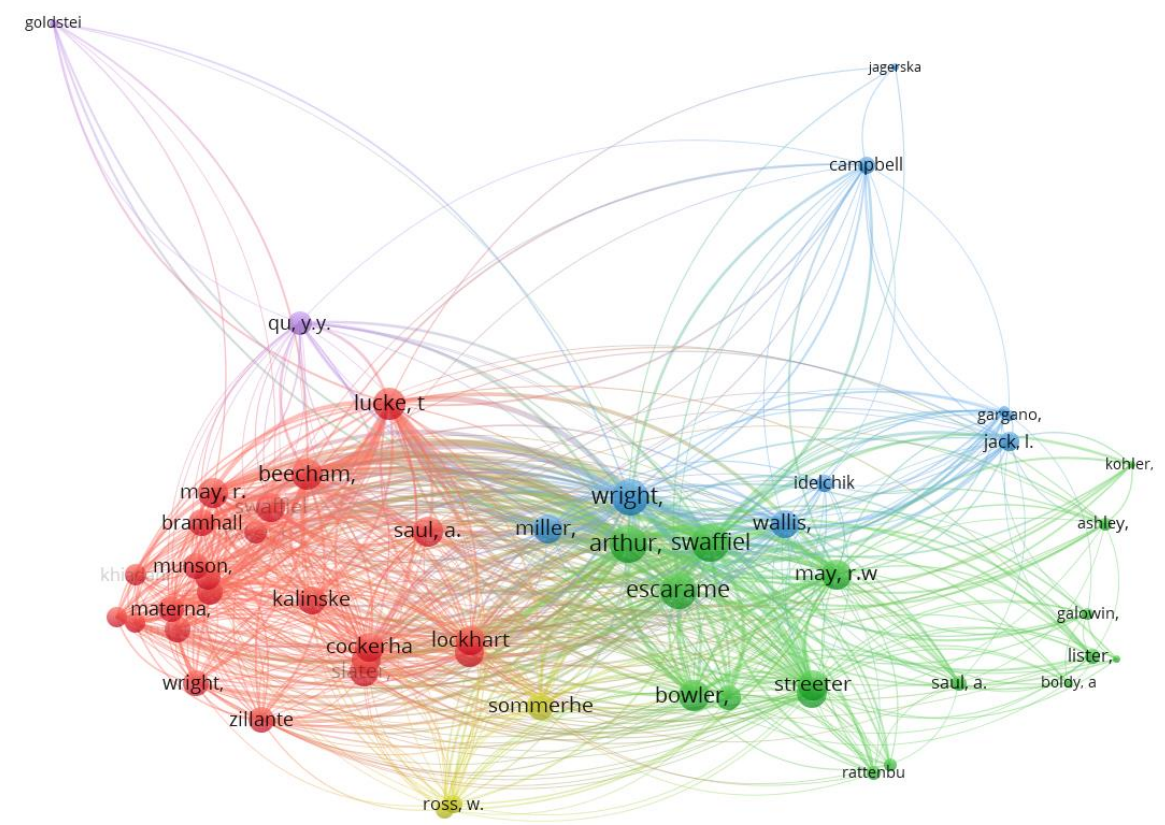

Fonte: Elaborada pelos Autores (2021). 
Na Tabela 2 estão demonstrados quais estudos foram mais relevantes para a construção teórica do assunto, e das 368 referências encontradas, 8 estudos foram citados mais de 3 vezes.

Tabela 2 - Trabalhos mais citados e quantidade de citações

\begin{tabular}{|c|c|c|}
\hline Id & Referências & $\begin{array}{l}\text { No de } \\
\text { citações }\end{array}$ \\
\hline 1 & $\begin{array}{l}\text { WRIGHT, G.B., SWAFFIELD, J.A., ARTHUR, S., The Performance Characteristics Of Multi- } \\
\text { Outlet Siphonic Rainwater Systems (2002) Building Services Engineering Research And } \\
\text { Technology. }\end{array}$ & 7 \\
\hline 2 & $\begin{array}{l}\text { ARTHUR, S., SWAFFIELD, J.A., Siphonic Roof Drainage System Analysis Utilising Unsteady } \\
\text { Flow Theory (2001) Building And Environment. }\end{array}$ & 6 \\
\hline 3 & $\begin{array}{l}\text { ARTHUR, S., SWAFFIELD, J.A., Siphonic Roof Drainage: Current Understanding (2001) } \\
\text { Urban Water. }\end{array}$ & 6 \\
\hline 4 & $\begin{array}{l}\text { ARTHUR, S., WRIGHT, G.B., SWAFFIELD, J.A., Operational Performance Of Siphonic Roof } \\
\text { Drainage Systems (2005) Building And Environment. }\end{array}$ & 6 \\
\hline 5 & $\begin{array}{l}\text { ARTHUR, S., SWAFFIELD, J.A., Siphonic Roof Drainage: The State Of The Art (2001) } \\
\text { Urban Water. }\end{array}$ & 5 \\
\hline 6 & $\begin{array}{l}\text { LUCKE, T., BEECHAM, S., Cavitation, Aeration And Negative Pressures In Siphonic Roof } \\
\text { Drainage Systems (2009) Building Services Engineering Research And Technology. }\end{array}$ & 5 \\
\hline 7 & $\begin{array}{l}\text { MAY, R.W.P., Escarameia, M., (1996) Performance Of Siphonic Drainage Systems For } \\
\text { Roof Gutters, , Report No. Sr463. Wallingford: Hr Wallingford }\end{array}$ & 3 \\
\hline 8 & $\begin{array}{l}\text { WRIGHT, G.B., ARTHUR, S., SWAFFIELD, J.A., Numerical Simulation Of The Dynamic } \\
\text { Operation Of Multi-Outlet Siphonic Roof Drainage Systems (2006) Building And } \\
\text { Environment. }\end{array}$ & 3 \\
\hline
\end{tabular}

Fonte: Elaborada pelos Autores (2021).

Outra avaliação realizada foi a de ocorrência de palavras-chave, sendo a rede apresentada na Figura 6.

Conforme avaliação de rede apresentada, a centralidade é formanda em torno dos termos "Drainage" e "Roofs" sendo estas as palavras chaves que mais aparecem na amostra, seguidas dos termos "Siphonic Roof Drainage", "Siphonic Roofs", "Rain", "Drainage Systems" que respectivamente compõem as palavras mais citadas. 
Figura 6- Mapa de Co ocorrência de palavras chaves

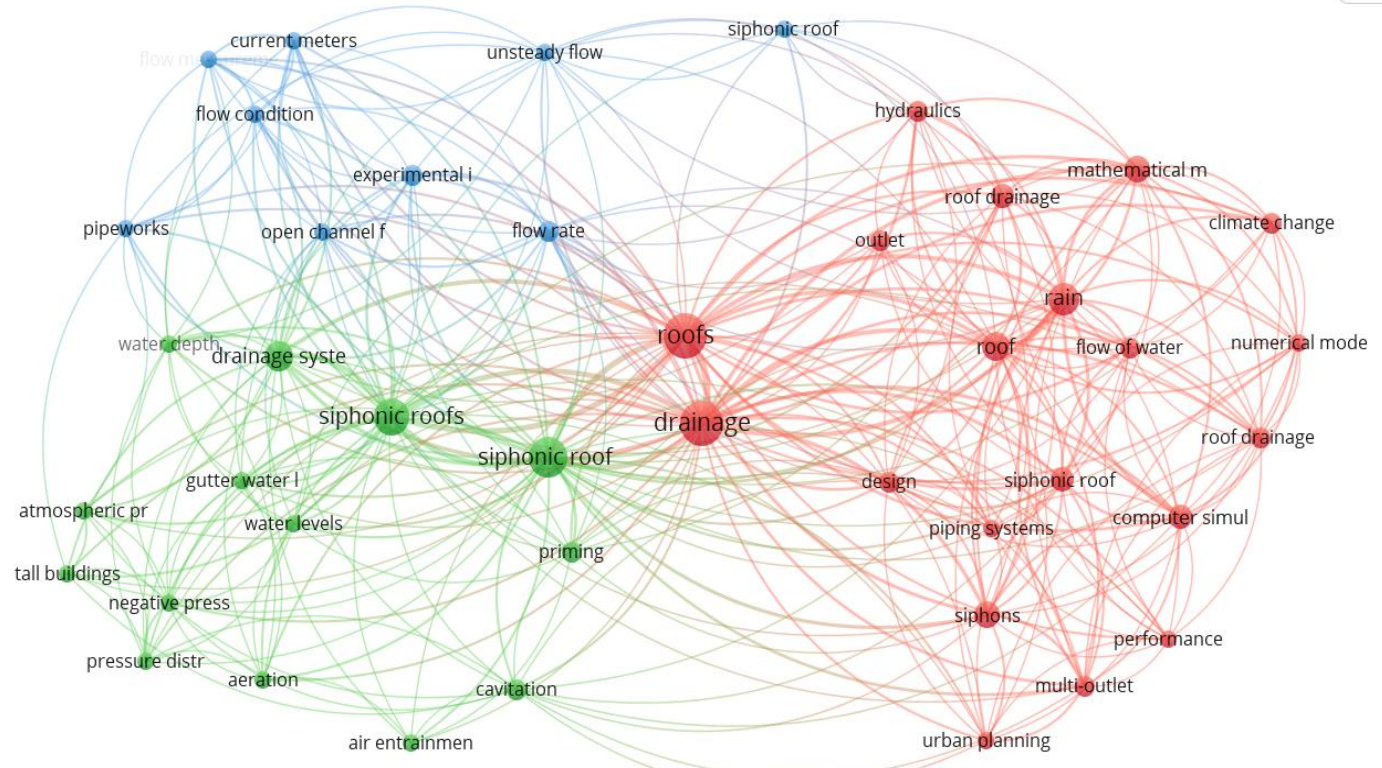

Fonte: Elaborada pelos Autores (2021).

Avaliou-se também o tipo de estudo realizado nos artigos, onde $54 \%$ foram reproduzidos em escala real e os estudos apresentados possuíam mais características experimentais / numéricas do que apenas teóricas, conforme classificação apresentada na Tabela 3.

Tabela 3 - Tipo de estudos realizados

\begin{tabular}{|l|c|}
\hline Classificação quanto ao tipo de estudo & Quantidade de estudos aderentes \\
\hline Experimental & 8 \\
\hline Experimental e Numérico & 10 \\
\hline Teórico & 6 \\
\hline Classificação quanto a escala aplicada & Quantidade de estudos aderentes \\
\hline Escala real & 13 \\
\hline Escala reduzida & 5 \\
\hline NA & 6 \\
\hline
\end{tabular}

Fonte: Elaborada pelos Autores (2021).

Por fim, foram separados e evidenciados, dentro dos trabalhos, os tópicos para melhor visualização sobre como estava sendo tratado o tema, sendo divididos em 7 categorias, conforme apresentado na Figura 7. 


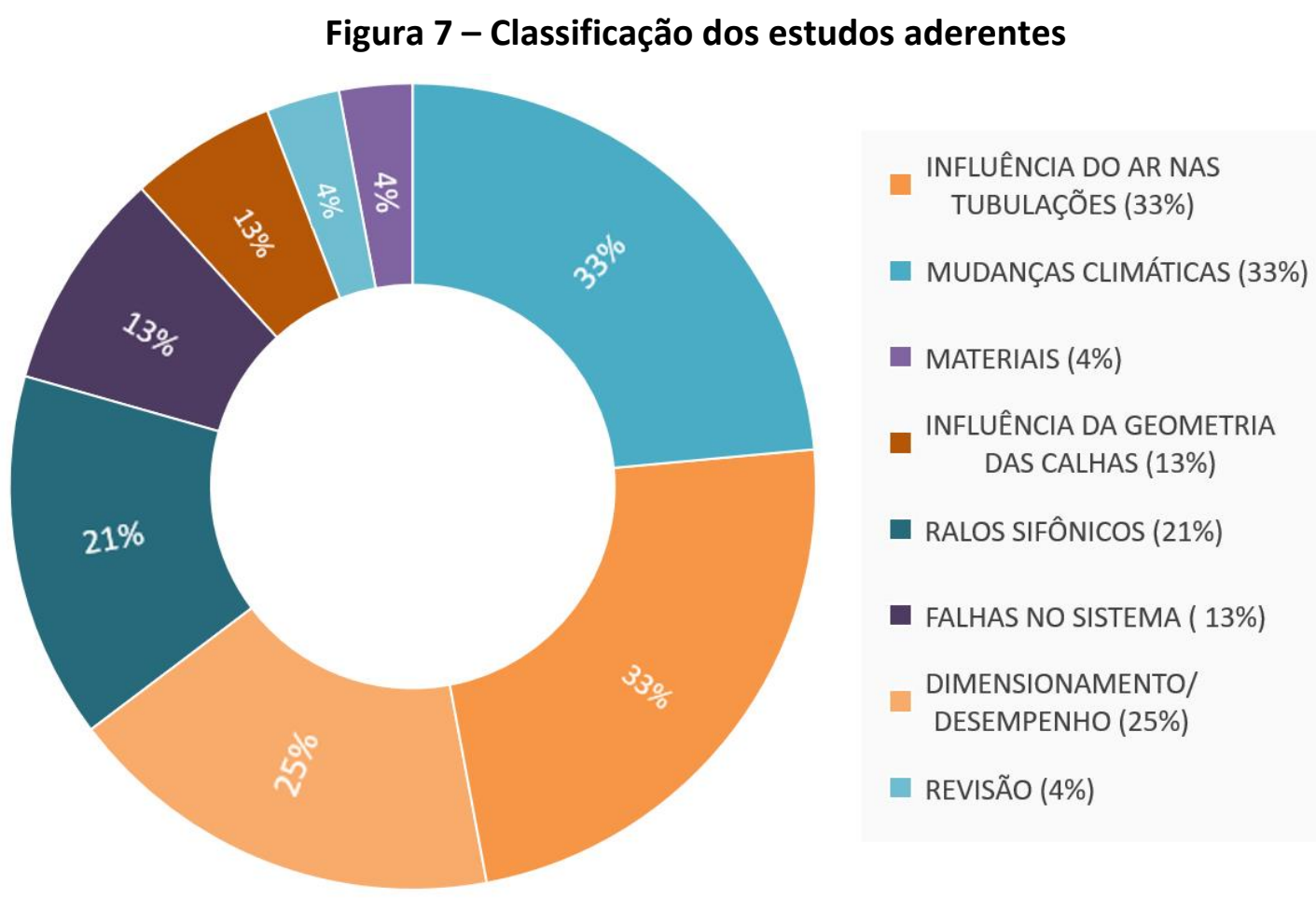

Fonte: Elaborada pelos Autores (2021).

As variáveis Clima, Geometria da Calha, Material da tubulação e Tomadas Sifônicas, foram debatidas em 67 \% dos trabalhos, a categoria influência do ar na tubulação também foi amplamente explanada sendo comentada em $33 \%$, enquanto que 0 dimensionamento/desempenho do sistema foi tratado em $25 \%$ dos estudos da amostra aderente.

Os estudos relacionados as falhas ocasionadas no sistema apareceram ainda em $13 \%$ da amostra e por fim $4 \%$ trouxeram uma revisão da literatura sobre o sistema.

Uma parcela de 33\% dos artigos, trataram da variável clima (MAY, 1997; ARTHUR; SWAFFIELD, 1999, 2001a, 2001b; ARTHUR; WRIGHT, 2007; LUCKE; BEECHAM, 2009, 2010a; BEECHAM; LUCKE, 2015).

A influência do ar na tubulação foi estudada por 33\% dos artigos aderentes (MAY, 1997; ARTHUR; SWAFFIELD, 1999; WRIGHT; SWAFFIELD, 2002; ARTHUR; WRIGHT, 2005; ARTHUR; WRIGHT; SWAFFIELD, 2005; LUCKE; BEECHAM, 2010a; BEECHAM; LUCKE, 2014; JACK; BEATTIE, 2014).

Tratativas sobre o dimensionamento dos ralos sifônicos ainda foram relatados por $21 \%$ dos trabalhos (MAY, 1997; LUCKE; BEECHAM, 2010a; JACK; BEATTIE, 2014; WRIGHT; SWAFFIELD ,2002; WRIGHT; SWAFFIELD; ARTHUR, 2002).

Outro parâmetro relevante para esta análise é a influência da geometria das calhas, aparecendo em 13\% dos estudos (ARTHUR; SWAFFIELD, 2001b; BEECHAM, S. BEECHAM; LUCKE, 2014). 
Explanações sobre o dimensionamento e desempenho do sistema, foi tratado em $25 \%$ dos estudos (MAY, 1997, WRIGHT; SWAFFIELD; ARTHUR, 2002; ARTHUR; SWAFFIELD, 2001; ARTHUR; WRIGHT, 2005; WRIGHT; JACK; SWAFFIELD, 2006; WRIGHT; ARTHUR; SWAFFIELD, 2006).

As falhas ocasionadas no sistema, apareceram em 13\% da amostra (CAMPBELL, 2014; LUCKE; BEECHAM, 2015; PIROGLU; OZAKGUL, 2016) e por fim, as análises quanto aos tipos de tubulação, se PVC ou PEAD, apareceram em apenas um estudo sendo 4\% (LUCKE; ARTHUR, 2011).

A amostra ainda trouxe uma revisão sobre o SSDAP, sendo 4\% do total (FUCIK; RUCKA,2019).

Dentro da amostra aderente, as questões relativas aos custos não apareceram com grande ênfase, apesar do SSDAP ser mais complexo e requerer uma manutenção mais acirrada que o sistema de drenagem convencional, nenhum dos artigos tratou do quesito da viabilidade econômica do sistema sifônico e, também, não foram encontradas, como objeto específico de estudo, ligações de todas as variações paramétricas com o custo global do sistema sinfônico.

As mudanças climáticas debatidas, tratavam-se apenas da região do Reino Unido, deixando a dúvida sobre o desempenho do sistema em locais com padrões de chuvas diferentes.

\section{CONCLUSÃO}

A partir da pesquisa realizada, foi possível cumprir o objetivo inicial proposto de conhecer a linha dos artigos que estão sendo publicados sobre o SSDAP. De um modo geral, os artigos foram publicados em periódicos com alto fator de impacto, o que manifesta a relevância e o potencial que possui a pesquisa envolvendo o SSDAP.

$\mathrm{Na}$ análise de rede (coautoria) observou-se pouca relação entre os autores dos documentos, a rede apresentou 7 grupos de colaboração em pesquisa, onde os autores mais citados são os pioneiros no assunto e os que possuem maior número de artigos.

Dos trabalhos experimentais, $54 \%$ foram reproduzidos em escala real, o que dá a entender que o estudo de caso é uma alternativa viável de modelo de pesquisa visto que, a maior parte da amostra de estudos aderentes utilizou deste modelo.

Partindo das lacunas observadas, pode-se sugerir como trabalhos futuros estudos que relacionem otimizações de projeto de SSDAP com base na viabilidade econômica, avaliação de desempenho dos SSDAP sob diferentes regimes pluviométricos, em especial no Brasil e estudos que abordem os custos gerais de implantação do SSDAP em detrimento dos sistemas ditos convencionais.

\section{REFERÊNCIAS}

ARTHUR S, SWAFFIELD, JA. Numerical modelling of the priming of a siphonic roof drainage system. Proceedings of CIBSE building services engineering researchand technology, 1999. Vol. 20, p. 83-91.

ARTHUR S, SWAFFIELD, JA. Siphonic roof drainage system analysis utilising unsteady flow theory. Building and Environment, 2001.Vol. 36, p. 939-948

ARTHUR, S. AND SWAFFIELD, J.A. Siphonic roof drainage: Current understanding, Urban Water, 2001. Vol. 3, p. 43-52. 
ARTHUR, S. AND WRIGHT, G. AND SWAFFIELD, J. Operational performance of siphonic roof drainage systems, Building and Environment, 2005. Vol. 40, pág. 788-796.

ARTHUR, S. AND WRIGHT, G.B. Recent and future advances in roof drainage design and performance, Building Services Engineering Research and Technology, 2005. Vol. 26, pág 337-348.

ARTHUR, S. AND WRIGHT, G.B. Siphonic roof drainage systems-priming focused design, Building and Environment, 2007. Vol 26, pág. 337-348.

ASSOCIAÇÃO BRASILEIRA DE NORMAS TÉCNICAS. NBR 10844: Instalações prediais de água pluvial. Rio de Janeiro, 1989.

BEECHAM, S. AND LUCKE, T. Air water flows in building drainage systems, Urban Water Journal, 2015. Vol. 12, pág. 455-467.

BEECHAM, S. AND LUCKE, T. Influence of channel geometry on water levels above siphonic roof outlets, Building Services Engineering Research and Technology, 2014. Vol. 35, pág. $83-98$.

CAMPBELL, D.P. Novel modulated flow, self configuring, siphonic roof drainage system, Building Services Engineering Research and Technology, 2014. Vol. 35, pág 349-361.

COSTA, C. H. A.; STAUT, S. L. S.; ILHA, M. S. O; Projeto de sistemas prediais hidráulicos sanitários com BIM: mapeamento da literatura, 11/2014, XV Encontro Nacional de Tecnologia do Ambiente Construído, Vol. 1, pp.2760-2769, Maceió, AL, Brasil, 2014.

DRESCH, A.; LACERDA, D. P.; ANTUNES JÚNIOR, J. A. V. Design Science Research: Método de pesquisa para avanço da ciência e tecnologia. Porto Alegre: Bookman, 2015.

FUCIK, D. AND RUCKA, J. Review of siphonic roof drainage systems, MM Science Journal, 2019, pág. 3683-3689.

JACK, L.B. AND BEATTIE, R.K. The impact of detritus accumulation on the performance of siphonic rainwater outlets, Building Services Engineering Research and Technology, 2014. Vol. 35, pág. $214-$ 232.

KITCHENHAM, B. (2004). Procedures for performing systematic reviews. Technical Report, Departament of Computer Science, Keele University, Keele. UK (TR/SE-0401), 28. ISSN : 1353-7776.

LUCKE, T. AND BEECHAM, S. Cavitation, aeration and negative pressures in siphonic roof drainage systems, Building Services Engineering Research and Technology, 2009. Vol. 30, pag 103-119.

LUCKE, T. AND BEECHAM, S. Aeration and gutter water levels in siphonic roof drainage systems, Building Research and Information, 2010. Vol. 38, pág. 670 - 685.

LUCKE, T. AND BEECHAM, S. Capacity loss in siphonic roof drainage systems due to aeration, Building Research and Information. Vol 38 - pág. 206-217.

LUCKE, T. AND ARTHUR, S. Plastic pipe pressures in siphonic roof drainage systems, Building Research and Information,2011, Vol. 39, pág. 79-92. 
LUCKE, T. AND BEECHAM, S. Alternative low-cost overflows for siphonic roof drainage systems: Proof of concept, Journal of Building Engineering, 2015, Vol. 2, pág. 9 - 16.

LUCKE, T. AND BEECHAM, S. and Qu, Y.Y. Estimating flow rates through individual outlets of siphonic roof drainage systems, Building Research and Information, 2016, Vol. 44, pág. 289-300.

MAY, R. W. P. The design of conventional and siphonic roof-drainage systems. Journal CIWEM, 11, Feb. 1997. Vol. 11, pág. $56-60$.

MAY, R. W. P. Design criteria for siphonic roof drainage systems. Wallingford: HRS, 2004. (Report SR 654).

PIROGLU, F. AND OZAKGUL, K. Partial collapses experienced for a steel space truss roof structure induced by ice ponds, Engineering Failure Analysis, 2016. Vol. 60, pág. $155-165$.

QU, Y.Y. AND LUCKE, T. AND BEECHAM, S. Measuring flowrates in partially-filled pipes in siphonic roof drainage systems, 15th International Flow Measurement Conference 2010, FLOMEKO 2010. Vol. 01, pág. $487-494$.

RUIZ, MILTON ARTUR; GRECO, OSWALDO TADEU; BRAILE, DOMINGO MARCOLINO. Fator de impacto: influência e influência no meio editorial, acadêmico e científico. Rev Bras Cir Cardiovasc, São José do Rio Preto, v. 24, n. 3, pág. 273-278, setembro de 2009.

NEES JAN VAN ECK AND LUDO WALTMAN (2009), VOSviewer: A Computer Program for Bibliometric Mapping, ERIM report series research in management Erasmus Research Institute of Management. Erasmus Research Institute of Management. Disponível em http://hdl.handle.net/1765/14841 . Acessado em 10/05/2021.

SIPHONIC ROOF DRAINAGE ASSOCIATION (SRDA) disponível em : http://www.siphonic-roofdrainage.co.uk/, acessado em 10/05/2021.

VAN ECK, N. J., \& WALTMAN, L. (2014). Visualizing bibliometric networks. In Y. Ding, R. Rousseau, \& D. Wolfram (Eds.), Measuring scholarly impact: methods and practice (pp. 285-320). London: Springer.

WRIGHT, G.B. AND SWAFFIELD, J.A. AND ARTHUR, S. Investigation into the performance characteristics of multi-outlet siphonic roof drainage systems, Global Solutions for Urban Drainage, 2002. Pág. 1-16.

WRIGHT, G.B. AND SWAFFIELD, J.A. The performance characteristics of multi-outlet roof drainage systems, Building Services Engineering Research and Technology, 2002. Vol. 23, Pág 127- 141.

WRIGHT, G.B. AND JACK, L.B. AND SWAFFIELD, J.A. Investigation and numerical modelling of roof drainage systems under extreme events, Building and Environment, 2006. Vol. 40, pág. 788 - 796.

WRIGHT, G.B. AND ARTHUR, S. AND SWAFFIELD, J.A., Numerical simulation of the dynamic operation of multi-outlet siphonic roof drainage systems, Building and Environment, 2006. Vol. 41, pág. 126-135. 